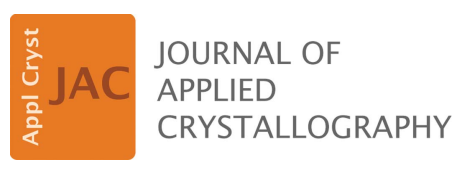

ISSN 1600-5767

Received 24 September 2015

Accepted 6 February 2016

Edited by A. Borbély, Ecole National Supérieure des Mines, Saint-Etienne, France

Keywords: X-ray orientation microscopy; fullfield imaging; grain microstructure; deformation twinning.
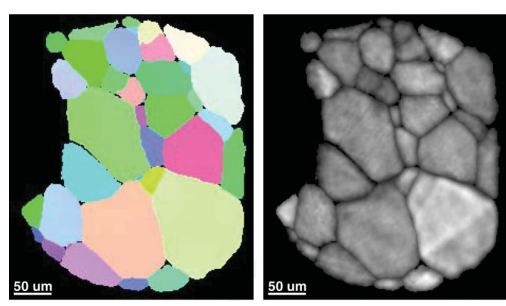

C 2016 International Union of Crystallography

\section{A feasibility study of full-field X-ray orientation microscopy at the onset of deformation twinning}

\author{
Nicola Viganò, ${ }^{\mathrm{a}, \mathrm{b}, \mathrm{c} *}$ Laura Nervo, ${ }^{\mathrm{b}, \mathrm{f}}$ Lorenzo Valzania, ${ }^{\mathrm{b}}$ Gaurav Singh, ${ }^{\mathrm{f}}$ Michael \\ Preuss, ${ }^{f}$ Kees Joost Batenburg ${ }^{d, c, e}$ and Wolfgang Ludwig ${ }^{a, b}$
}

${ }^{\mathbf{a}}$ Université de Lyon, INSA Lyon, CNRS, MATEIS UMR5510, France, ${ }^{\mathbf{b}}$ ESRF Grenoble, France, ${ }^{\mathbf{c}} \mathrm{iMinds}-\mathrm{Vision}$ Lab,
Univeristy of Antwerp, Belgium, ${ }^{\mathbf{d}} \mathrm{CWI}$, Amsterdam, Netherlands, ${ }^{\mathbf{e}}$ Universiteit Leiden, Netherlands, and ${ }^{\mathbf{f}}$ School of
Materials, University of Manchester, UK. ${ }^{*}$ Correspondence e-mail: nicola.vigano@esrf.fr

Three-dimensional X-ray orientation microscopy based on X-ray full-field imaging techniques such as diffraction contrast tomography is a challenging task when it comes to materials displaying non-negligible intragranular orientation spread and/or intricate grain microstructures as a result of plastic deformation and deformation twinning. As shown in this article, the optimization of the experimental conditions and a number of modifications of the data analysis routines enable detection and three-dimensional reconstruction of twin lamellae down to micrometre thickness, as well as more accurate three-dimensional reconstruction of grains displaying intragranular orientation spreads of up to a few degrees. The reconstruction of spatially resolved orientation maps becomes possible through the use of a recently introduced six-dimensional reconstruction framework, which has been further extended in order to enable simultaneous reconstruction of parent and twin orientations and to account for the finite impulse response of the X-ray imaging detector. The simultaneous reconstruction of disjoint orientation domains requires appropriate scaling of the scattering intensities based on structure and Lorentz factors and yields threedimensional reconstructions with comparable density values for all the grains. This in turn enables the use of a global intensity-guided assembly procedure and avoids problems related to the single-grain thresholding procedure used previously. Last but not least, carrying out a systematic search over the list of known twin variants (forward modelling) for each of the indexed parent grains, it is possible to identify additional twins which have been left undetected at the previous stage of grain indexing based on diffraction spot peak positions. The enhanced procedure has been tested on a $1 \%$ deformed specimen made from a Ti-4\% Al alloy and the result has been cross-validated against a twodimensional electron backscatter diffraction orientation map acquired on one of the lateral sample surfaces.

\section{Introduction}

Four-dimensional (time-lapse) multi-modal observation of polycrystalline materials microstructures as they evolve as a function of strain and/or temperature can provide valuable benchmark data for adjustment and validation of image based numerical simulations of polycrystalline aggregates. The process of deformation twinning encountered in structural materials with hexagonal crystal structure like $\mathrm{Mg}, \mathrm{Ti}$ and $\mathrm{Zr}$ is an example of industrial relevance (forming of critical components in aerospace and nuclear applications) where the predictive capabilities of theoretical models for microstructure evolution are not yet satisfactory and where refinement and extension of existing models could potentially be facilitated by direct comparison of model predictions with experimental observations.

However, time-lapse observations of deformed and/or twinned microstructures by means of three-dimensional 
synchrotron X-ray diffraction techniques are a challenging task. Established methods suitable for orientation mapping in deformed microstructures, like differential aperture X-ray microscopy (Larson et al., 2002), X-ray diffraction tomography (Bonnin et al., 2014; Hayashi et al., 2015), and three-dimensional X-ray diffraction and high-energy diffraction microscopy (Poulsen, 2012; Li \& Suter, 2013), use focused X-ray beams and are based on two- or three-dimensional acquisition procedures. Owing to limitations in instrumentation and detector technology these scanning procedures currently suffer from time overheads, which limit the ultimate acquisition rate and lead to compromises in terms of sample volume and/or time steps that can be recorded in the course of a typical synchrotron beamtime. Tomographic full-field microstructure mapping techniques like X-ray diffraction contrast tomography (DCT) (Ludwig et al., 2009; Reischig et al., 2013) require only a single rotational scan. Given the longer exposure time for individual images (as compared to one- or twodimensional focused beams) these techniques are less affected by the above-mentioned technical limitations. As a consequence, relatively large $\left(10^{6}-10^{7}\right.$ voxel) sample volumes can be characterized in a fraction of the time currently reported for two- and three-dimensional scanning approaches. ${ }^{1}$

However, until recently the reconstruction approach behind DCT has lacked the ability to address materials with nonnegligible intragranular orientation gradients. With the introduction of a six-dimensional reconstruction framework (Viganò et al., 2014, 2016) this technique can now be extended to the case of moderately deformed materials displaying intragranular orientation spreads of up to a few degrees. In this paper we present an extension of this full-field X-ray orientation imaging approach, tailored for the specific challenges related to reconstruction of materials having undergone moderate levels (up to a few percent) of plastic deformation, partly accommodated by the formation of deformation twins.

\subsection{Challenges related to the reconstruction of deformation} twins

Deformation twins are typically observed as disc-like crystal domains with thickness and size distributions spanning from nanometre to micrometre length scales (Christian \& Mahajan, 1995). Their crystallographic orientation is related to the parent orientation by a well defined pair of rotation axis and rotation angle around one of the symmetry axes of the crystal (see Appendix $A$ for a brief recap of the calculation of twin variant orientations given the orientation of the parent grain and the twin axis and angle pair of the twinning mode under consideration). This means that all Bragg reflections asso-

\footnotetext{
${ }^{1}$ A typical value at ID11, ESRF, would be $400^{3}$ voxels within $2 \mathrm{~h}$, corresponding to a gain of a factor 10 and 100 in acquisition time as compared to currently reported acquisition times for slice beam and point beam scanning techniques, respectively. With progress in instrumentation, the slicing and scanning approaches may become competitive in time resolution. Since these scanning approaches offer less convoluted and hence easier to analyse diffraction data, adaptations of the global (six-dimensional) optimization framework to these acquisition modes can be envisaged.
}

ciated with scattering vectors colinear to the said twin rotation axes will be shared between parent and twin and project to the same position and diffraction spot on the detector. As a result, while most of the reflections recorded on the detector will be independent projections of the two domains, a subset of them will be shared. If these shared reflections are to be included in a joint reconstruction procedure, where parent and twin domains are reconstructed simultaneously (as envisaged in this work), the diffracted intensities have to be scaled properly on the basis of structure and Lorentz factors. ${ }^{2}$

The high aspect ratio and small scattering volume of deformation twins result in diffraction signals which can be orders of magnitude weaker than that of the corresponding parent grain. Moreover, owing to the high aspect ratio, the local intensity received on the high-resolution screen varies as a function of projection direction and systematically drops to the noise level for projections close to the direction of the twin plane normal. These systematic absences are in turn a great challenge for the indexing and the tomographic reconstruction algorithm, which suffer from the poor angular coverage. In addition, particularly projections in directions perpendicular to the plane normal ('edge-on' views, observed as intense lines) suffer from blurring due to the limited impulse response of the high-resolution imaging detectors employed in this type of near-field diffraction imaging experiment. This together with the partial-volume effect (drop of reconstructed intensity for objects with thickness below the pixel size of the reconstruction) results generally in substantially broadened twin reconstructions and lower reconstructed intensities compared to the parent grains.

Last but not least, deformation twins are triggered and accompanied by other types of plastic deformation (dislocation glide), giving rise to increasing levels of disorder and intragranular orientation spread with increasing deformation. Even moderate plastic deformations in the range of one percent can already result in the formation of grain substructures and orientation spreads of the order of one degree. The resulting three-dimensional diffraction blob volumes are deformed grain projections that violate the parallel projection assumption of conventional (single-orientation) DCT, which can no longer produce accurate grain shape reconstructions in this case.

The two obvious experimental solutions to alleviate some of the above-mentioned problems are improving the signal over noise ratio by reducing the background on the detector and increasing the illumination on the sample, as described in \$3.1.

In terms of data analysis strategy the approach pursued in this work was to create the infrastructure for simultaneous reconstruction of the parent and twin, using an upgraded version of the recently introduced six-dimensional reconstruction framework (Viganò et al., 2014, 2016), as described in the next section. Furthermore, we show that a basic search

\footnotetext{
${ }^{2}$ Since the relative volume fraction of parent and twin are not known beforehand, a joint reconstruction of parent and twin can no longer rely on the renormalization of the diffraction blob intensities used in previous implementations of the reconstruction algorithm where all the identified and segmented reflections would be renormalized to the same intensity.
} 
over all possible twin variants (derived from the known parent grain orientations in the sample volume) can help to reveal additional twins, left undetected by the indexing procedure.

\section{Method}

The usual setup of a near-field diffraction experiment, when using a monochromatic beam, consists of a rotation stage where the sample is positioned, and as the sample rotates by angle $\omega$, it gives rise to diffracted beams each time the Bragg condition is met for one of the grains. Some of those diffracted beams will intersect the high-resolution imaging detector positioned a few millimetres downstream of the sample and give rise to diffraction spots, which, in the absence of intragranular orientation spread, correspond to two-dimensional projections of the three-dimensional grain volume. After diffraction spot segmentation and indexing based on Friedel pairs ( $h k l$ and $\overline{h k l}$ reflections of the same grain observed for $\omega_{0}$ and $\omega_{0}+180^{\circ}$ ) the three-dimensional grain structure can be reconstructed by means of iterative tomographic reconstruction techniques. The reader interested in details concerning the setup, acquisition procedures and initial processing steps like segmentation, Friedel pair matching and indexing of nearfield X-ray diffraction data is referred to Ludwig et al. (2009) and Reischig et al. (2013).

In the presence of non-negligible intragranular orientation spread, the parallel projection assumption used in conventional (three-dimensional) DCT is violated. Each of the suborientations present in a grain is associated with a slightly different projection geometry, and the diffraction signal associated with a given Bragg reflection is observed as a distorted three-dimensional diffraction volume, which then takes the name of diffraction 'blob'. It is parametrized by two spatial coordinates $u$ and $v$ (detector pixel coordinates) and a rotation angle $\omega$ (image number). ${ }^{3}$

It has been recently shown that these types of diffraction data can be inverted by switching to a six-dimensional description, in which case maps of local (intragranular) orientation can be produced using suitable optimization and regularization techniques (Viganò et al., 2014, 2016). Unlike the case of three-dimensional DCT, where only one single (grain average) orientation is considered per grain, the sixdimensional formulation, 6D-DCT, assigns a discrete threedimensional orientation distribution to each of the real-space voxels inside a grain. In order to compare the six-dimensional scalar field reconstruction output with conventional threedimensional vector field representations of the orientation field, we transform the six-dimensional output into a threedimensional orientation map, by assigning the average orientation (centre of mass of the local orientation subspace) to each real-space voxel. Since this six-dimensional framework

\footnotetext{
${ }^{3}$ Another parametrization of the diffraction 'blob' positions on the detector, instead of $(u, v)$, is given in ch. 3 of Poulsen (2004), where the aperture (Bragg) angle between the incoming beam and the diffracted beam is called $2 \theta$, and the angle between the projection of the rotation axis and the line connecting the intercepts of the direct beam $\left(u_{0}, v_{0}\right)$ and the diffracted beam $(u, v)$ on the detector is called $\eta$.
}

intrinsically accounts for the distortion of the diffraction blob volumes it results in improved shape reconstructions compared to the single-orientation (three-dimensional) reconstruction framework.

In order to adapt the six-dimensional framework to the case of deformation twins we further extend the previously introduced concept of 'cluster' reconstructions (Viganó et al., 2016) to the case of disjoint orientation domains (applicable to other cases of grain substructures with a priori known orientation relationships or grain neighbourhoods), thereby enabling simultaneous reconstruction of parent and twin domains from experimental raw data. As mentioned earlier, for this to become possible the previous concept of diffraction spot intensity renormalization has been abandoned and replaced by an appropriate scaling of projection matrix, taking intensity variations between different $h k l$ reflections owing to variations in structure factor and Lorentz factors explicitly into account. Apart from alleviating problems with segmentation of parent and twin reconstructions, the simultaneous reconstruction has the added benefit that additional (shared) reflections between parent and twin domain can be included in the reconstruction process. Moreover, our model of diffraction image formation has been enhanced and now includes blurring effects caused by the finite impulse response of the X-ray imaging detectors employed in this type of experiment.

\subsection{Mathematical formulation}

As set out by Viganò et al. (2014), the reconstruction space, if elastic strain is supposed to be negligible, is a six-dimensional space $\mathbb{X}^{6}=\mathbb{R}^{3} \otimes \mathbb{O}^{3}$ obtained by the outer product of Cartesian real space and three-dimensional orientation space. The representation of this six-dimensional space is discretized by choosing a sampling that can be viewed in two equivalent ways. The first and most intuitive sampling would consist of creating a three-dimensional orientation sampling for each of the real-space sampled positions. This would give rise to a collection of orientation distributions functions for each of the real-space voxels. Although this representation may be intuitive, it is not very computationally efficient, and we preferred to use a collection of identical real-space volumes, each of which is associated with a different sampled point in the orientation space.

We also point out that, while the parent grain and its twins are reconstructed within the same bounding box in real space, their bounding boxes are instead separate in orientation space, and that, within limits of memory occupation, it is possible to include several subvolumes (e.g. the different twin variants) in orientation space.

If now we assume that the image formation can be described by kinematic diffraction and that we can neglect photoelectric absorption and extinction effects, the forward projection of the grain volumes can be represented as a linear operator:

$$
\mathbf{A x}=\mathbf{b},
$$


where $\mathbf{x}$ is a vector containing $N P$ elements, representing the scalar 'scattering power' for each of the sampled volume elements in the six-dimensional position-orientation space $\left(N P=n^{3} \times k p^{3}\right.$ for the case of a regular sampling over a cubeshaped subvolume with grid length $n$ in real space and $k$ disjoint subvolumes in orientation space, each sampled with grid length $p$ ). Each line of the projection matrix $\mathbf{A}_{S \times N P}$ contains the contribution of the six-dimensional volume elements to a given detector pixel and the vector $\mathbf{b}_{S}$ holds a list of measured pixel intensities, specified by their $(u, v, \omega)$ coordinates in the three-dimensional diffraction image stack. $S$ corresponds to the total number of detector pixels in the $M=k M_{h k l}-c$ diffraction blob volumes relating to the joint set of parent and twin reflections recorded during the scan. Here $M_{h k l}$ represents the total number of reflections intercepting the detector, which is usually of the order of a few tens up to 100 , and $c$ represents the small number of reflections shared between the parent and twin orientations.

If the elements of $\mathbf{x}$ are arranged as a succession of threedimensional real-space volumes, each representing one of the sampled orientations, and $\mathbf{A}$ as an array of $M \times P$ projection matrices, each one describing the projection geometry for one of the $M$ reflections intercepted by the detector, the vector $\mathbf{b}$ will be composed of $M$ blocks corresponding to contiguous three-dimensional subvolumes (i.e. the aforementioned diffraction blobs), spread throughout the entire stack of detector images:

$$
\left(\begin{array}{ccc}
\mathbf{A}_{11} & \cdots & \mathbf{A}_{1 P} \\
\vdots & \ddots & \vdots \\
\mathbf{A}_{M 1} & \cdots & \mathbf{A}_{M P}
\end{array}\right) \quad\left(\begin{array}{c}
\mathbf{x}_{1} \\
\vdots \\
\mathbf{x}_{P}
\end{array}\right)=\left(\begin{array}{c}
\mathbf{b}_{1} \\
\vdots \\
\mathbf{b}_{M}
\end{array}\right) .
$$

The beauty of equation (2) is, however, limited by the fact that it is not suitable for the actual reconstruction. The number of diffraction blobs that fall on the detector $(M \simeq 30-100)$ is limited, and these spots are affected by noise and overlaps, so equation (1) is a heavily underdetermined and perturbed equation system which needs to be rewritten as a minimization problem.

Some additional constraint and prior should be added to the minimization functional in order to promote solutions that are compliant with the known physical properties of the microstructure under investigation. In the case of a moderately deformed metal sample, imaged at $1.5 \mu \mathrm{m}$ resolution, as presented in this study, two physically justified prior assumptions are the positiveness and sparsity of the six-dimensional solution vector. Indeed, we only expect a limited number of 'active' orientations inside a given real-space volume element, and this behaviour could be enforced by adding an $l_{1}$ penalty term to the optimization functional, as proposed by Viganò $e t$ al. (2014). Here we have used an alternative formulation, minimizing the spatial variability of the reconstructed intensity $^{4}$ as proposed recently by Viganó et al. (2016):

\footnotetext{
${ }^{4}$ The intensity value obtained from projecting the six-dimensional space onto the three-dimensional real-space grid can be interpreted as the local 'scattering power' of the material.
}

$$
\begin{gathered}
\mathbf{x}^{*}=\underset{\mathbf{x}}{\operatorname{argmin}}\|\mathbf{A x}-\mathbf{b}\|_{2}+\lambda\|(|\nabla S \mathbf{x}|)\|_{1}, \\
\text { subject to: } \mathbf{x} \geq \mathbf{0},
\end{gathered}
$$

where $S$ is the operator that sums all the orientation components from both the parent and the twins orientation spaces for each real-space voxel, and the $l_{1}$ norm over the absolute value of the gradient is the total variation operator (Candès \& Romberg, 2005; Sidky et al., 2012).

In this work however, while we formally keep the same functional because the general idea is still completely valid, we had to adapt many details under the cover of equation (3), to be able to deal successfully with the intrinsic nature of the twin reconstructions.

From physics we know that the parent and its twins occupy exclusive but also contiguous regions in the real-space reconstruction domain. This means that they do not overlap but also that there is no empty spacing between the two domains. To force a homogeneous real-space volume with sharp boundaries at the grain edges and transitions at the parent-twin boundaries that are also homogeneous, the most natural choice is a total variation minimization over the sum of all the orientation components of the real-space voxels, which means that this time the sum is over two disjoint regions in orientation space.

As reported by Viganò et al. $(2014,2016)$, the algorithm that solves this optimization problem was derived from a specific class of algorithms called Chambolle-Pock, slightly modified to cope with the new type of functional, as described by Sidky et al. (2012).

In the next sections we will also analyse other peculiar details of equation (3), which mark the progress achieved compared to the work of Viganó et al. (2016).

\subsection{Scaling of the projection matrix}

From the mathematical point of view, the renormalization comes from the decomposition of equation (1), as follows:

$$
\mathbf{A} \mathbf{x}=\mathbf{C} \tilde{\mathbf{A}} \mathbf{x}=\mathbf{C} \sum_{i}^{N} \tilde{\mathbf{A}}_{i} \mathbf{x}=\mathbf{b},
$$

where the matrix $\mathbf{C}$ is a diagonal matrix, which predicts the scattering intensities for each of the reflections, and the matrix $\tilde{\mathbf{A}}$ only contains the geometric projection coefficients to the detector. The renormalization procedure simply pre-multiplies both sides of equation (4) as

$$
\tilde{\mathbf{A}} \mathbf{x}=\sum_{i}^{N} \tilde{\mathbf{A}}_{i} \mathbf{x}=\mathbf{C}^{-1} \mathbf{b}=\tilde{\mathbf{b}}
$$

where now $\tilde{\mathbf{b}}$ are the renormalized diffraction blobs. The advantage of this new formulation is a better conditioning of the problem, the diagonal of the matrix $\tilde{\mathbf{A}}$ being more homogeneous.

Finally, while most of the reflections are not shared between a parent and its twins, and so the projection to the diffraction blobs happens separately, for what concerns the shared reflections, the forward projection and back projection should 
happen simultaneously for both the parent and the twins belonging to the same diffraction blobs.

\subsection{Point spread function and blurred reconstructions}

Near-field diffraction imaging experiments are typically performed with detector systems based on CCD cameras, coupled via visible light optics to a transparent luminescent screen that converts the incoming X-rays into visible light. Owing to the large interaction volume of X-rays and the diffraction limit of the visible light microscope optics, common designs of these systems are known to be limited in spatial resolution to values of typically a few times the effective detector pixel size. The performance of such an optical system can be described by its impulse response (point spread function, PSF) and/or the modulation transfer function. For X-ray imaging systems, both depend on details of the experimental conditions (i.e. energy of X-ray beam, type and thickness of scintillator crystal, visible light optics etc.). In the present study the point spread function was approximated by a symmetric, translationally invariant Gaussian profile with a full width at half-maximum of about 2 pixels. (As further discussed in $\$ 5$ a more realistic description of the PSF would depend on the detector region and on the incidence direction of the photons on the scintillator screen.)

If we now take equation (5), where the scattering intensity has been translated into a renormalization of the diffraction blobs, and so the forward model is expressed in a pure geometrical form, it can be modified to include the PSF blurring as follows:

$$
\mathbf{F} \tilde{\mathbf{A}} \mathbf{x}=\mathbf{F} \sum_{i}^{N} \tilde{\mathbf{A}}_{i} \mathbf{x}=\tilde{\mathbf{b}}
$$

where the matrix $\mathbf{F}$ is the operator responsible for applying the PSF to the forward-projected images. Practically speaking this translates into a simple convolution between the forwardprojected diffraction blobs and the PSF specific to each of them.

When trying to invert the forward operator $\mathbf{F} \tilde{\mathbf{A}}$ by first premultiplying it by its transpose, we have to take into account that the PSF is generally speaking not symmetric, and so the adjoint of $\mathbf{F}$ should be taken, resulting in

$$
\tilde{\mathbf{A}}^{\mathrm{T}} \mathbf{F}^{\dagger} \mathbf{F} \tilde{\mathbf{A}} \mathbf{x}=\tilde{\mathbf{A}}^{\mathrm{T}} \mathbf{F}^{\dagger} \tilde{\mathbf{b}}
$$

where the superscript $\uparrow$ stands for adjoint (complex conjugate transpose).

Finally, these considerations about the PSF were always to be considered negligible in the previous work using the sixdimensional approach, the lack of orientation-space resolution being the major limiting factor in the reconstruction quality. However, in the case of twin reconstructions, where the twins can be sometimes less than $1 \mu \mathrm{m}$ thick and the detector pixel resolution is above $1 \mu \mathrm{m}$, even moderate effects like the PSF blurring of very few pixels could heavily impact on the quality of reconstruction of these thin regions of the grains.

\subsection{Finding twins from the parent orientation}

For the reasons explained in $\$ 1.1$, deformation twins are intrinsically difficult to index from near-field diffraction data using conventional indexing schemes based on diffraction spot peak positions. Nevertheless, the detection ratio can be improved by a systematic search for intensity (forward modelling) in the background-corrected raw images, starting from the known orientation of the successfully indexed parent grains. In the following we restrict our discussion to one specific twinning mode, predominant for the material studied in this work, but the suggested strategy can be applied to any other known twinning modes.

The Ti-4 wt \% Al alloy studied in this work has a hexagonal crystal structure with a $c / a$ ratio smaller than that for optimum close packing. When deformed in compression in the direction parallel to the initial rolling direction, the most frequently observed twinning mode for this type of material is one known as a tensile twin of type 1 (TT1) (Christian \& Mahajan, 1995). This twinning mode corresponds to a rotation of about $85^{\circ}$ around $[10 \overline{1} 0]$, and the hexagonal crystal symmetry allows for six different variants of the same type.

Given the parent orientation, the orientation matrix of the twin variants can be precomputed (see Appendix $A$ ) and from this the approximate twin diffraction spot peak position $[(u, v, \omega)$ coordinates in the detector space] can be determined. Since twins are confined within the volume of the parent grain we can restrict the search of the twin reflections to diffraction blobs that do not exceed the size of the parent grain. At the same time we allow for blobs that have a high variability in aspect ratio, as expected from the disc-like morphology of deformation twins.

However, the actual twin orientation might be slightly different from the theoretically predicted orientation (derived from the average orientation of the parent grain), and the position of the twin inside the parent grain is not known $a$ priori. So it is convenient to express the detector region for the search of the twin reflections in terms of the $(\theta, \eta, \omega)$ coordinates, allowing for small deviations from the nominal values, in order to account for these uncertainties.

\section{Experiment and material}

A sample of $2.5 \mathrm{~mm}$ length and a diameter of $0.5 \mathrm{~mm}$ was extracted by electro-discharge machining parallel to the cylinder axis and rolling direction of a larger compression sample made from a binary $\mathrm{Ti}-4 \mathrm{wt} \% \mathrm{Al}$ alloy. The larger specimen had been deformed in compression to about $1 \%$ plastic strain in the direction parallel to the cylinder axis, i.e. parallel to the former rolling direction. The preferential orientation of the $\langle c\rangle$ axis in the direction perpendicular to the cylinder axis and loading direction promotes tensile twinning during compression loading as the $\langle c\rangle$ axis is strained in tension (Hertzberg, 1989). The grain size of the material determined by the linear intercept method was $70 \mu \mathrm{m}$.

In a parallel study on the same specimen an analysis of twin nucleation criteria based on a three-dimensional recon- 
structed sample volume containing 400 grains and about 60 twins revealed a correlation of the propensity for twinning with grain size and a clustering of twinned grains in neighbourhoods with favourable prismatic slip transmission conditions (Nervo, 2015).

\subsection{DCT data acquisition}

The experiment was performed on the three-dimensional XRD instrument of the materials science beamline ID11 of the European Synchrotron Radiation Facility (ESRF). The incident beam was monochromated to $60 \mathrm{keV}$ and focused to dimensions of approximately $0.2 \times 0.5 \mathrm{~mm}$ by means of a set of compound refractive lenses. Under the experimental conditions described above, the focusing results in about a factor of 20 increase in flux density at the sample position compared to the configuration without lenses. This increase was found to be a crucial requirement for successful detection of deformation twins which are known to nucleate as disc-like tapered lamellae of (initially sub-) micrometre thickness.

The cylindrical sample was mounted with its cylinder axis parallel to the rotation axis of the diffractometer, and the focused incident beam was confined by slits to a section of $0.15 \mathrm{~mm}$ in the vertical and $0.6 \mathrm{~mm}$ in the horizontal direction. The sample-to-detector distance was set to $8 \mathrm{~mm}$ and the diffraction images were recorded on a CCD based and light optic based high-resolution detector system featuring a $50 \mu \mathrm{m}$ thick transparent luminiscent screen prepared from an Eudoped gallium gadolinium garnet single crystal (GGG) (Smith (2006) and a long-working-distance visible light microscope objective $(10 \times$, natural aperture $=0.23)$. The resulting effective pixel size was $1.4 \mu \mathrm{m}$. As mentioned earlier, increasing the flux density by at least one order of magnitude compared to acquisitions in materials with comparable grain size is a prerequisite for successful indexing and reconstruction of deformation twins down to micrometre thickness.

The introduction of the absorber significantly reduces the background signal on the scintillation screen and allows us to raise the exposure time and hence diffraction spot intensities by the inverse of the $\mathrm{X}$-ray transmission $(\sim 20$ times in the case of $0.5 \mathrm{~mm}$ of Ta at $60 \mathrm{keV}$ ).

For the DCT scan a total of 3600 images with $2.5 \mathrm{~s}$ exposure time were recorded during a synchronized continuous $360^{\circ}$ motion of the air-bearing diffractometer stage. A second scan with $0.2 \mathrm{~s}$ exposure time and 360 projections but without the additional absorber was recorded right after the DCT scan, maintaining the relative position of sample and detector. These data were used for tomographic reconstruction of the $\mathrm{X}$-ray attenuation coefficient, serving as a mask in the process of assembling the individual grain volumes into the common sample volume.

\subsection{EBSD analysis}

The electron backscatter diffraction (EBSD) characterization was performed on a cross section parallel to the axis and loading direction of the cylindrical sample. The sample was embedded into epoxy resin and then a layer of approximately
$200 \mu \mathrm{m}$ was removed using 4000 grit $\mathrm{SiC}$ grinding paper. For best Kikuchi pattern acquisition, the sample was polished with oxide polishing suspension ( $80 \%$ colloidal silica $+20 \% \mathrm{H}_{2} \mathrm{O}_{2}$ by volume) for $\sim 2 \mathrm{~h}$ and etched repeatedly with Kroll's reagent. The surface finish was further improved by a final vibratory polishing with oxide polishing suspension in a Buehler VibroMet 2 machine. To overlap the three-dimensional region scanned in DCT, a $600 \times 90 \mu \mathrm{m}$ area was selected and scanned using a step size of $0.4 \mu \mathrm{m}$ in a field emission gun scanning electron microscope (FEI QUANTA 650) equipped with a NordLYS EBSD detector. The EBSD mapping was performed at an operating voltage of $20 \mathrm{kV}$ with a working distance of $13 \mathrm{~mm}$. The AZtec software (Oxford Instruments, Abingdon, UK) was used for EBSD data acquisition. Post analysis of the EBSD data, including inverse pole figure map generation and selection of the axis-angle misorientation pair for determination of the active twin mode, was performed using the HKL Channel 5 software (Oxford Instruments, Abingdon, UK).

\section{Results}

From inspection of the EBSD map (Fig. 1), it appears that most of the twins intersecting the sample surface correspond to tensile twins $\left(85^{\circ}\right.$ rotation around $\left.[10 \overline{1} 0]\right)$.

In the common surface area shared between the EBSD and DCT acquisition, two grains have developed (sub-)micrometre thickness twins of this type. One of them (Fig. 2a) was successfully identified during the indexing step of the DCT data reduction process (Ludwig et al., 2009; Reischig et al., 2013), whereas two other domains could only be detected during a systematic search, using the prediction (forward simulation) of twin orientation variants calculated from the orientation of the parent grain (Fig. $2 b$ ), as can be seen in Appendix $A$.

Fig. 3 shows a slice though the reconstructed three-dimensional intensity volume (i.e. the sum of all orientation-space components of the six-dimensional reconstruction output) of the grain rendered in Fig. 2(a), illustrating the difficulty of correctly reconstructing the precise shape and volume of these sub-micrometre thickness twins domains. In fact, while the thickness of twin lamellae should be smaller than a single voxel, in the reconstruction they extend over several voxels, and while they should present a reconstruction intensity that is comparable to the average intensity in the parent grain, it is possible to see from the scale bars in Fig. 3 that the twins present much lower intensity values (see $\$ 5$ for a detailed discussion of the origins of these discrepancies).

In this article we focus on the $150 \mu \mathrm{m}$-high subregion of the full sample highlighted in the EBSD map in Fig. 4(a). As mentioned earlier, the six-dimensional output of the algorithm is converted into a three-dimensional orientation field in order to enable direct comparison with the EBSD orientation map. This transformation is justified and the comparison meaningful if the size of the EBSD interaction volume and the voxel size of the DCT reconstruction are comparable to each other and if the size of the volume elements and the orientation 


\section{research papers}

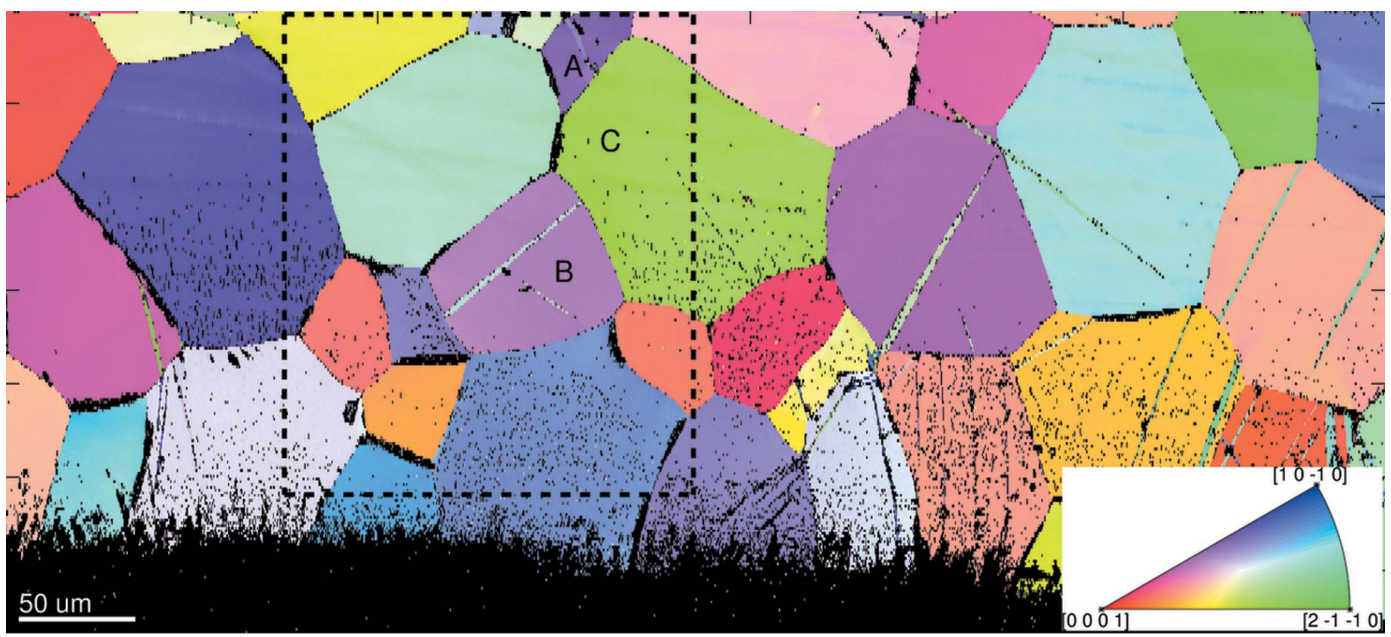

(a)

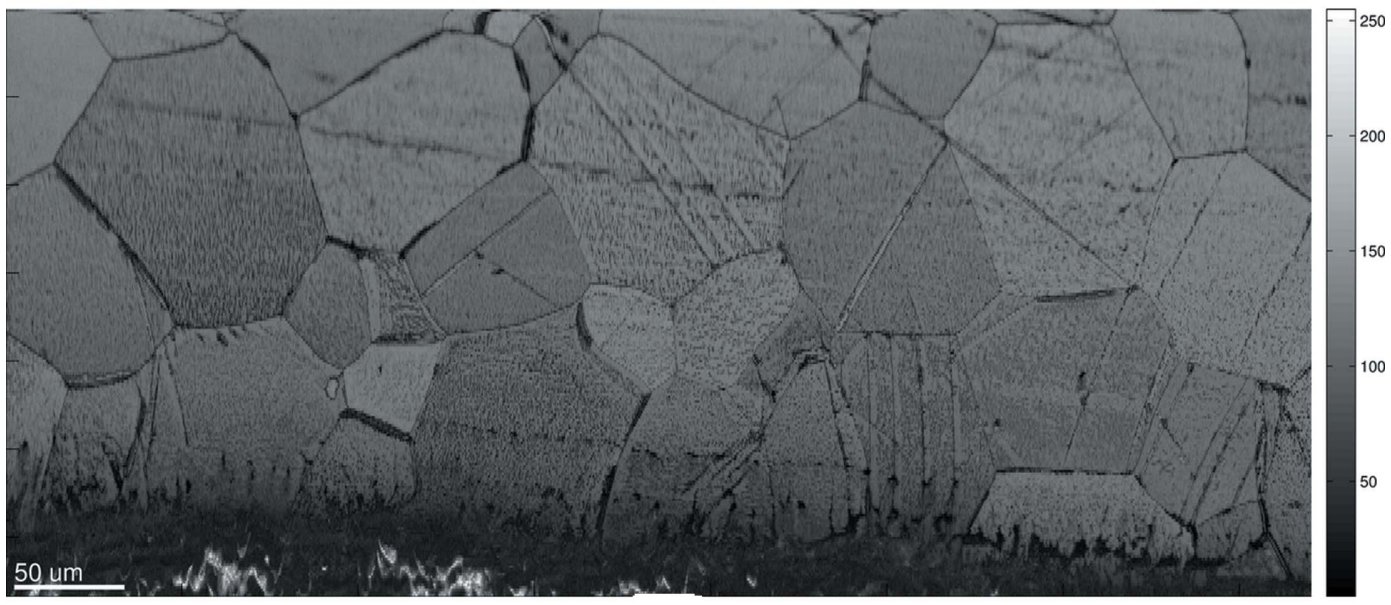

(b)

Figure 1

(a) Orientation map of a longitudinal sample cross section parallel to the rolling direction, recorded by EBSD (IPF colour code). The dashed lines indicate the sample subvolume characterized in the DCT scan. (b) Quality map (band contrast) of the map in $(a)$ on the byte scale. Deformation twins are clearly discernible on the EBSD map, while slip traces are more easily discerned on the quality map (e.g. inside the grain labelled with the letter C).

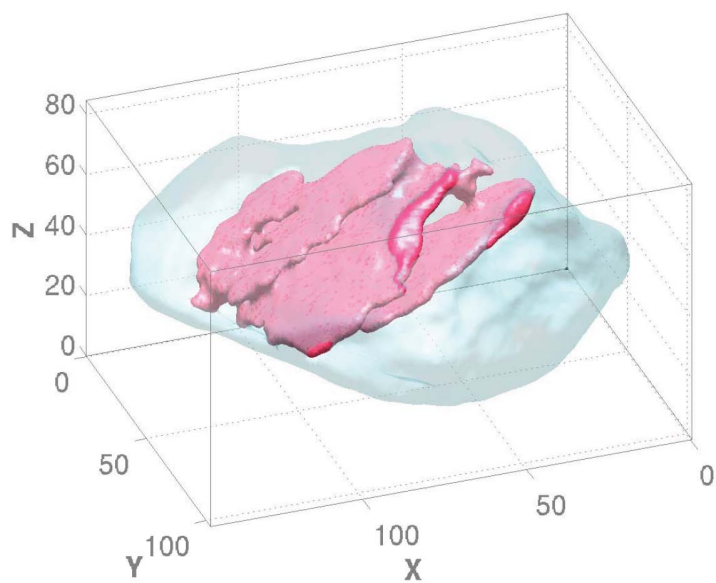

(a)

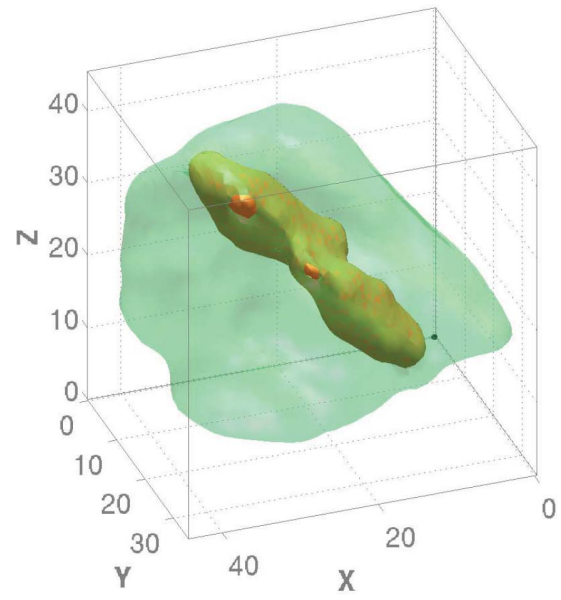

(b)

Figure 2

Three-dimensional rendering of two twinned grains, as reconstructed from 6D-DCT. The grains correspond to the grains labelled A and B, respectively, in Fig. 1 and they have been rendered with transparency in order to reveal the three-dimensional shape of the embedded twins. The voxel size is $1.4 \mu \mathrm{m}$, and the size of the bounding boxes is $103 \times 145 \times 83$ voxels and $34 \times 44 \times 44$ voxels, respectively. The slightly irregular reconstructed shape of the twin lamellae is partly due to problems in segmenting the six-dimensional tomographic reconstructions obtained from the very low signal-to-noise ratio projection data. 
gradients are sufficiently small to contain only a limited range of active orientations.

Fig. 4(b) shows the 6D-DCT reconstruction close to the surface region shown in Fig. 4(a). The quantitative analysis of average orientations shows good agreement between the two modalities, whereas the shape reconstruction shows some differences, most visible for the smaller grains. We attribute these differences to the combination of the following factors: (i) the intensity and sensitivity at the surface of DCT reconstructions are highly affected by the detector PSF, which tends to blur and diminish the signal coming from these regions; (ii) twin domains of sub-voxel-size thickness are subjected to the partial volume effect and result in very weak signal, especially at the sample surface; (iii) the actual sample surface is slightly curved and Fig. 4(b) corresponds to the orientations observed on a shifted (2 pixels into the volume) copy of this surface (see $\S 5$ for further explanations concerning this relative shift).

In Fig. 2 both twinned grains from Fig. 4 were rendered and the previous statement (ii) about the differences between Figs. 4(a) and 4(b) becomes more clear, since it can be seen that the reconstructed twinned region does not fully reach to the surface of the grain but extends very close to it. Figs. 4(c) and $4(d)$, in fact, show how the grain structure and especially the shape of the twins evolve while going deeper and deeper into the volume, confirming the previous statement.

These findings are consistent with our previous observation (Viganó et al., 2016) indicating that even with the upgrade to a six-dimensional reconstruction model it remains difficult to reliably segment grain reconstructions from the outermost $1-2$ voxel thickness surface layer of a grain/sample reconstruction. If the X-ray characterization is carried out after the EBSD characterization as in the present case, the required relative shift into the DCT volume (for more consistent grain segmentation) and the possible curvature of the EBSD surface give rise to discrepancies that render the direct comparison of the results (Figs. $4 a$ and $4 b$ ) problematic.

In addition, an even thinner twinned region is visible in Fig. 5(a). Its thickness appears to be smaller than the acquisition step size of the EBSD map $(0.4 \mu \mathrm{m})$, and it was not possible to identify this second twin with the DCT acquisition conditions described in this paper.

In Fig. 5 we take a closer look at the smooth deformation gradients in one of the grains of the analysed surface region and compare the results between the EBSD map and the 6DDCT reconstruction. The analysed grain was unfortunately damaged by a few scratches during the polishing phase, but it

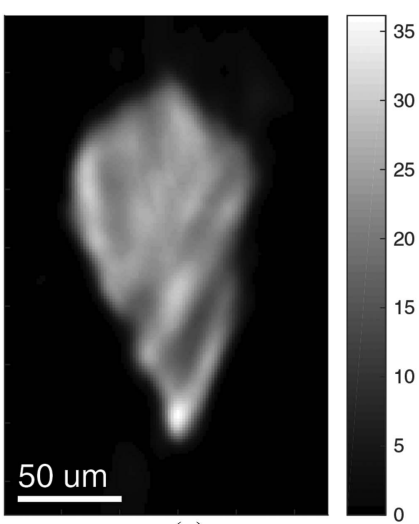

(a)

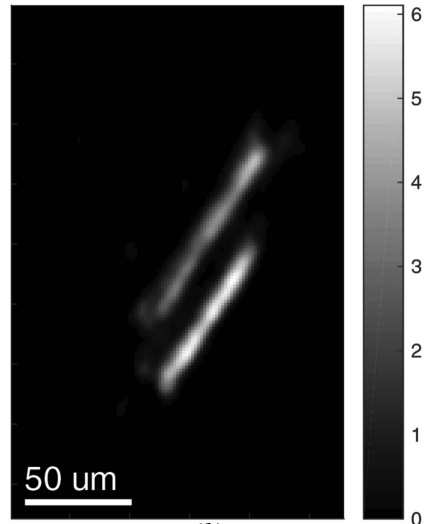

(b)

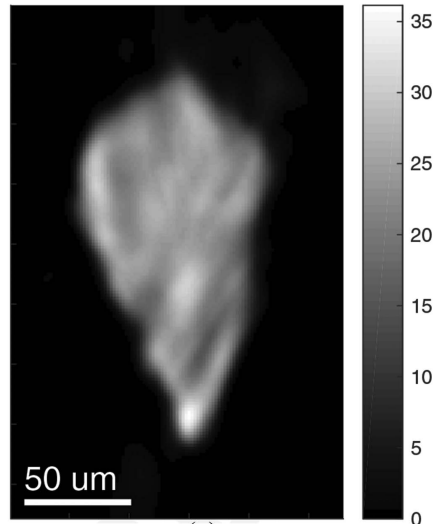

(c)

Figure 3

Slice 134 in the $Z Y$ plane through the reconstruction volume of Fig. 2(a): (a) the parent reconstruction, $(b)$ the twin reconstruction and $(c)$ the sum of the two. The presence of effects like PSF and local strain clearly affects the reconstruction of the twin, which, as a consequence, exhibits a much lower intensity than the parent and a thickness of several voxels.

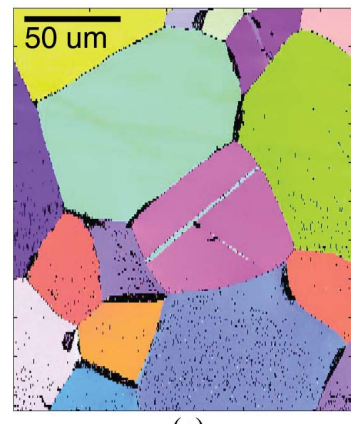

(a)

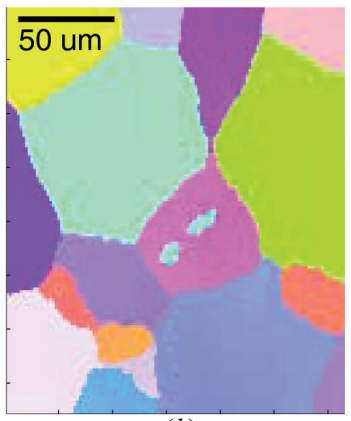

(b)

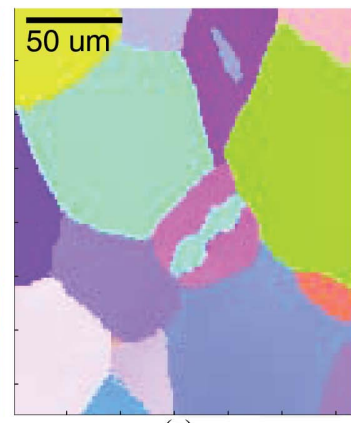

(c)

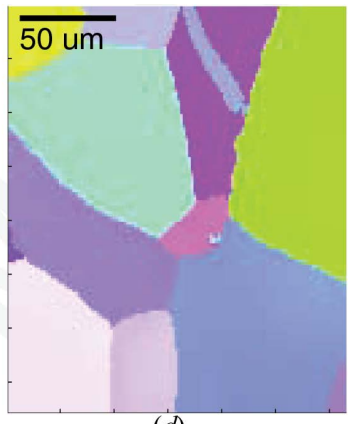

(d)

Figure 4

Comparison of orientation maps of the sample surface (IPF colour coding) obtained by (a) EBSD and $(b)$ 6D-DCT at $3.5 \mu \mathrm{m}$ from the surface. Subfigures $(c)$ and $(d)$ show the sample grain structure at 13.3 and $27.3 \mu \mathrm{m}$, respectively, from the surface. 


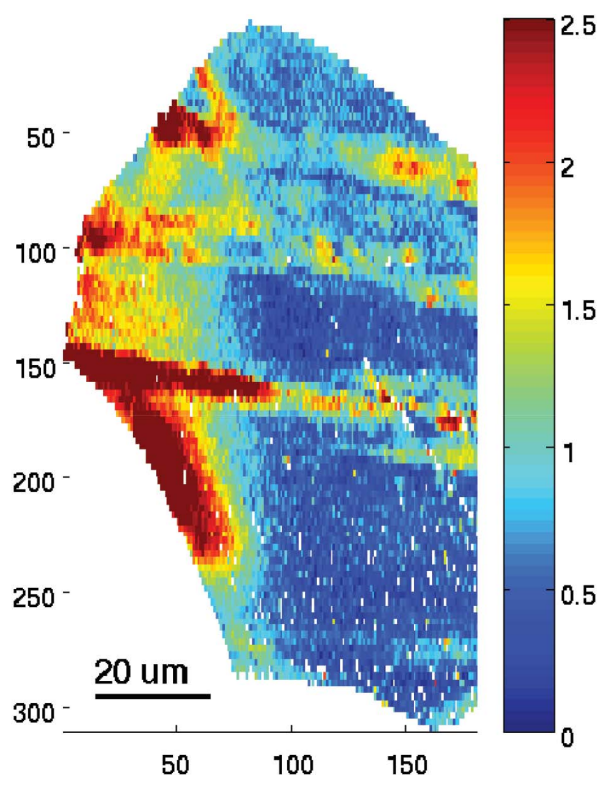

(a)

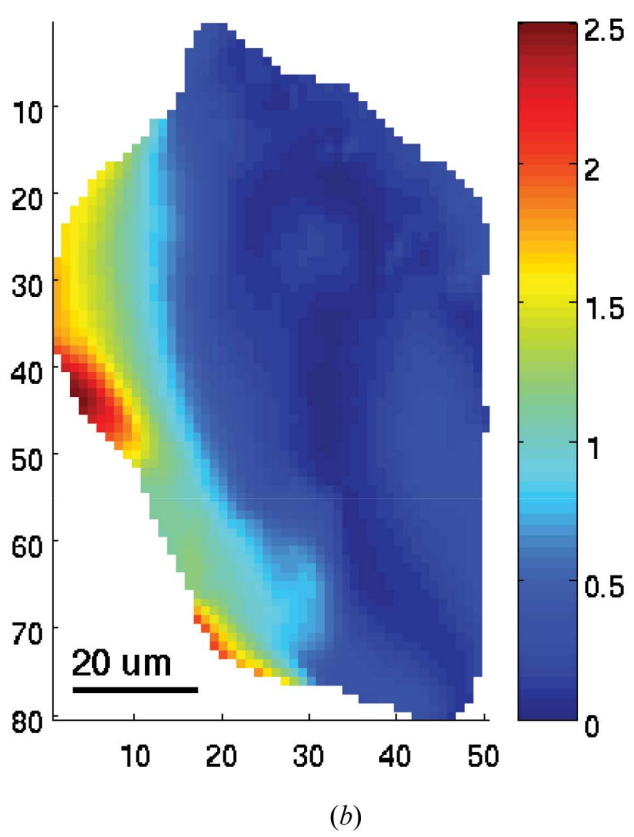

(b)

Figure 5

Intragranular misorientation of the grain labelled as $\mathrm{C}$ on the surface shown in Fig. 1. The EBSD $(a)$ and DCT $(b)$ maps have voxel sizes of 0.4 and $1.4 \mu \mathrm{m}$, and therefore the numbers of voxels shown on the figure axes are different, while the scale bars are similar.

is also the one that shows the strongest and most easily recognizable orientation gradient among the reconstructed surface grains. Indeed, if we exclude the obvious deformation due to the scratches in Fig. 5(a), and remember that the same

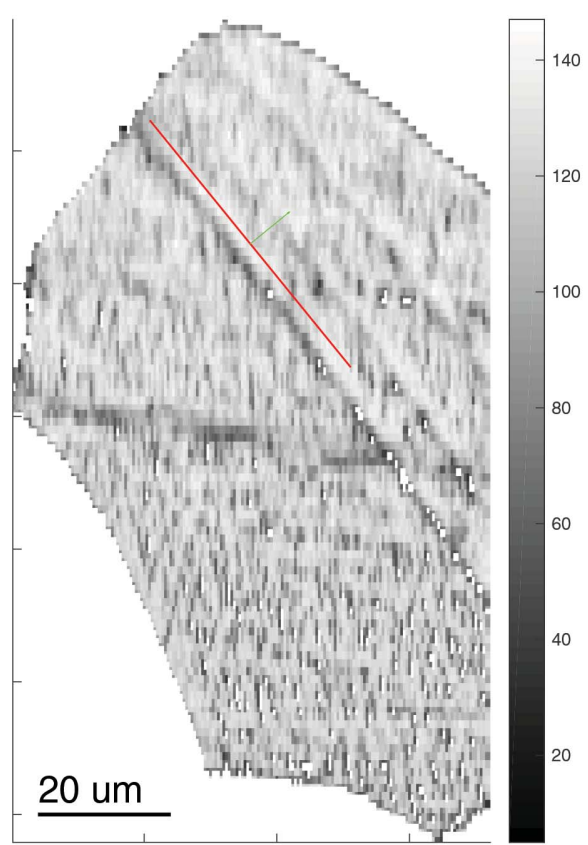

(a)

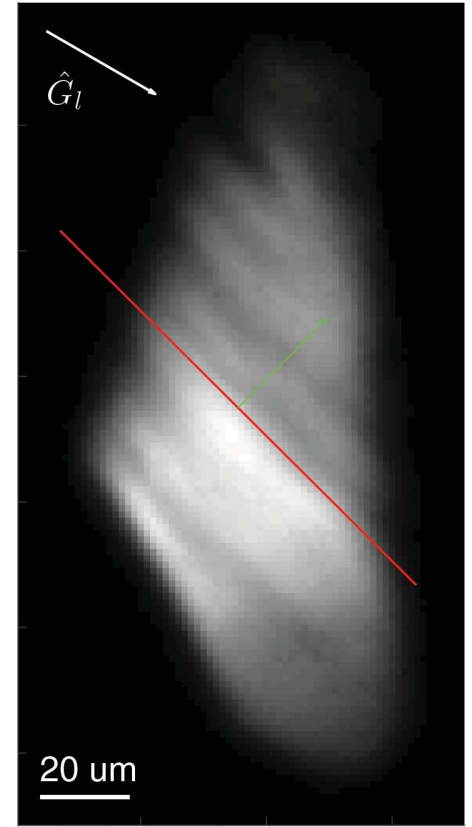

(b)
Figure 6

(a) EBSD band contrast map for the grain depicted in Fig. 5, revealing the presence of slip bands. The inclination of these bands corresponds to the trace of the (prismatic) [0110] plane in the crystal, represented by the red line, while the green arrow is the projection of its plane normal. (b) The integrated $\langle 01 \overline{1} 1\rangle \mathrm{X}$-ray projection topograph of the illuminated three-dimensional grain volume, showing topographic orientation contrast. The red line is the intersection of the (prismatic) [0110] plane with the detector, while the green arrow is again the projection of the corresponding plane normal. limitations introduced before still apply, a similar deformation gradient can be observed in both Figs. 5(a) and 5(b).

What is not completely obvious from Fig. 5 is the presence of slip bands inside the grain. This is instead noticeable both from the band contrast map obtained for the EBSD measurements (Figs. $1 b$ and $6 a$ ) and from the diffraction spots recorded by DCT (Fig. 6b). However, in the 6D-DCT reconstruction, just like in the EBSD measurement, the local misorientations in the vicinity of these slip bands were too small to be clearly distinguished.

Finally, in Fig. 7 we can see a comparison of the assigned average orientation for each real-space voxel and the total scattering intensity assigned by the six-dimensional reconstruction algorithm. The similar average intensities among all the grains (the variations are around a few percent) are a result of the renormalization of the diffraction blobs discussed in $\$ 2.2$, where instead of renormalizing all the spots belonging to the same grain to a common arbitrary value, we decided to renormalize them on the basis of theoretically predicted scattering intensities for each crystallographic family and different Lorentz factors. The main outcome of this procedure is that these intensities are now more physically meaningful and can be compared among different grains, in order to solve grain conflicts. This in turn results in better accuracy of the grain boundary and triple junction positions, and yields grain maps that show a higher coverage of the sample volume, even before any type of dilation is applied. 


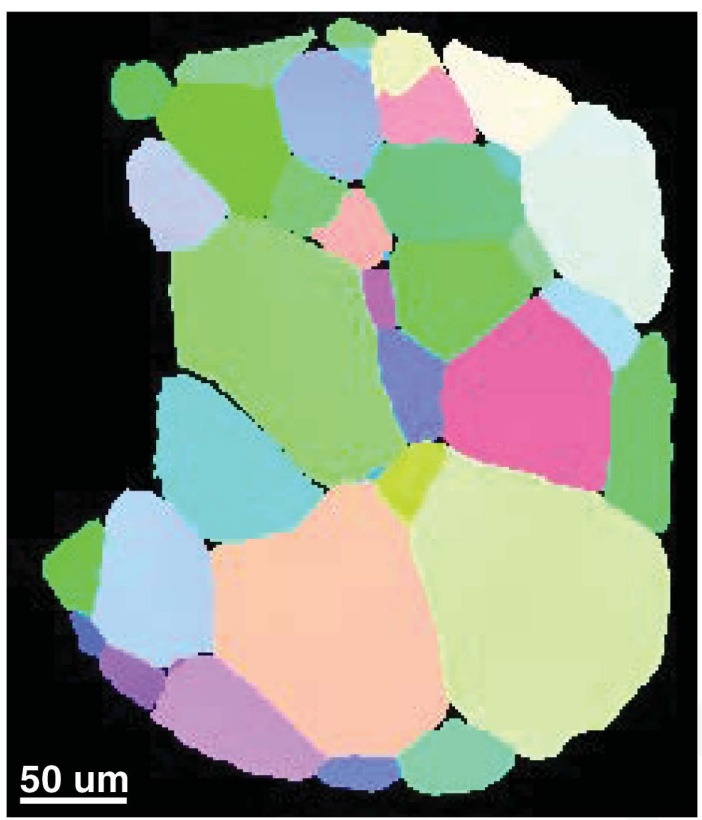

(a)

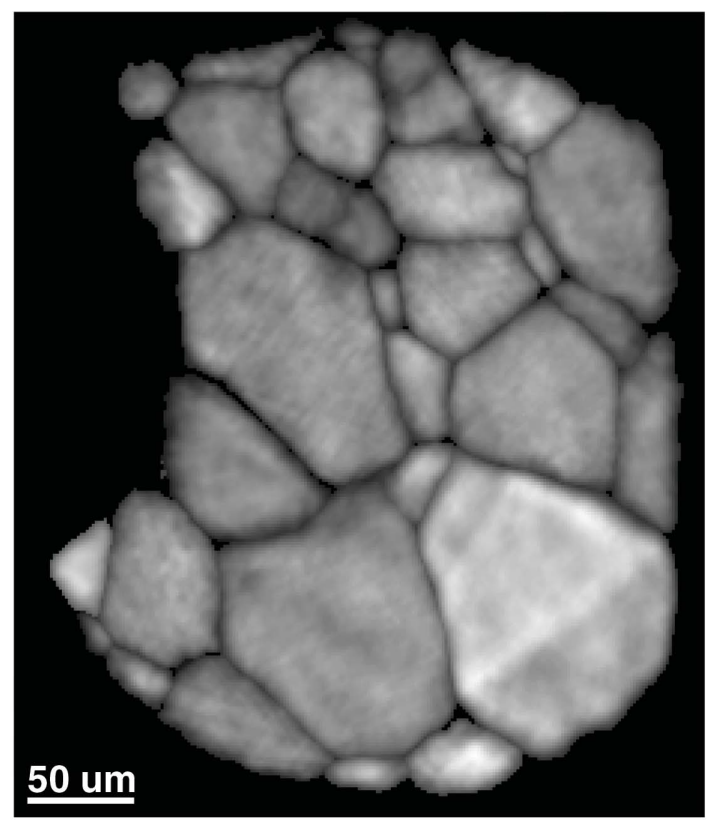

(b)

Figure 7

Slice through the full reconstructed volume. (a) Inverse pole figure colouring of the grain orientations, using the colour coding from the legend in Fig. 1(a). (b) Intensity assigned by the 6D-DCT reconstruction in each real-space voxel.

Some remaining deviations in the average intensity can be observed for some of the grains in Fig. 7. The physical origin of these variations remains to be clarified.

\section{Discussion}

The high aspect ratio of deformation twins gives rise to large (up to two orders of magnitude and higher) variations of the signal received on individual pixels of the high-resolution imaging detector as a function of rotation angle. In order to raise the signal of unfavourable reflections (i.e. those with the direction of the diffracted beam close to the direction of the surface normal of a twin lamella) above the background, and hence assure uniform angular coverage of the projections from a twin domain, it is mandatory to increase the photon flux density at the sample position far beyond the level usually employed for an equiaxed grain structure. With the flux provided by state-of-the-art undulator insertion devices and the use of tunable X-ray focusing optics (Vaughan et al., 2011) the total scanning time for the $150 \times 500 \mu \mathrm{m}$ sample subvolume was $2.5 \mathrm{~h}$. In order to extend the detection limit towards even smaller (thinner) twins, a further increase of the photon flux density is required. This will become available with the one to two order of magnitude increase in brilliance predicted for the next generation of low-emittance storage rings. Alternatively, one may consider switching to onedimensional focused line beam illumination mode, compromising ultimate time resolution but enabling different reconstruction strategies [forward modelling (Suter et al., 2006; Li \& Suter, 2013) or global five-dimensional optimization approaches for simultaneous reconstruction of the entire sample cross section] which may outperform the current six-dimen- sional framework based on grain by grain reconstruction at higher levels of deformation.

In the present work we have incorporated a rather crude estimate of the point spread function modelled as a rotationally symmetric Gaussian profile. Further improvement can be expected from a measurement of the actual profile for different angles of incidence on the detector and introduction of an orientation-dependent PSF, reproducing the directional asymmetry caused by the parallax of the diffracted beam impinging with different angles $\left(2 \theta_{\text {Bragg }}, \eta\right)$ on the scintillator screen with finite thickness.

The oversimplified model of the detector impulse response together with the partial volume effect known from conventional absorption tomography are supposed to be the principal reasons for the lower reconstructed densities of voxels corresponding to twinned regions.

Fig. 4(b) shows some discrepancies in the grain shapes as determined by EBSD and our full-field reconstruction approach. These discrepancies are partly related to the fact that the X-ray orientation map has been extracted from a virtual surface situated approximately two pixels $(3 \mu \mathrm{m})$ below the actual sample surface, as determined from the absorption volume.

As mentionned earlier, we notice some gradient in the reconstructed intensity values over the first few pixels of a grain volume. The presence of this gradient renders greyscale segmentation of these volumes problematic. The small offset of the surface from which the orientation values have been extracted helps to overcome this segmentation problem but starts to introduce some visible shifts, especially for boundaries intersecting the sample surface at a small angle.

Moreover, the sample surface is slightly curved (an artefact from the mechanical polishing procedure). We have deter- 
mined the actual shape of the surface from the phase contrast reconstruction of the sample and we have extracted the orientations (Fig. 4b) from the intersection of this curved surface with the (2 pixel shifted) three-dimensional orientation volume.

Within the limits of computer memory available, the formalism used in this paper allows for simultaneous reconstruction of arbitrary sets of disjoint orientations occupying a common volume in real space. By extending the real-space volume to a cluster of neighbouring grains, it is therefore possible to use the same framework for simultaneous reconstruction of three-dimensional grain neighbourhoods. This procedure would circumvent the step of grain shape determination by means of grey level segmentation of the intensity volumes of the individual grains, and it can be expected that such combined reconstructions will result in an overall improvement of the three-dimensional grain shape reconstruction accuracy, similar to that observed for deformation twins presented in this study.

The current reconstruction approach is still essentially a two-step process of grain indexing based on scattering vectors determined from diffraction spot peak positions. Owing to increasing levels of spot overlap, the concepts behind grain indexing are known to fail gradually for increasing levels of plastic deformation. On the other hand, by repeated characterization of a sample volume at increasing levels of plastic deformation it should be possible to seed the reconstruction of the next step with the information from orientation maps reconstructed at previous step(s), thereby extending the range of plastic deformation that can be addressed with the current approach beyond the level of the feasibility study presented in this work. It can be expected that additional diffraction data acquired on a far-field detector with a pixel size comparable to or larger than the grain size and higher quantum efficiency would allow for identification of twins at early stages during deformation since the entire twin volume would typically project onto individual pixels, thereby removing the systematic intensity variations as a function of the angle between the twin lamella and the diffracted beam direction observed on the near-field detectors. Using the centre of mass position and orientation determined from far-field data, it would be possible to use these twin 'seeds' in the joint sixdimensional reconstruction of the corresponding parent grains.

The example shown in Fig. 6 confirms the visibility of topographic orientation contrast related to the presence of slip bands in grains that are favourably oriented for prismatic slip. Unfortunately, the sensitivity of the 6D-DCT reconstruction is not high enough to reveal the faint lattice rotations giving rise to topographic orientation contrast observed in diffraction spots with favourably oriented scattering vectors.

We anticipate that, by using a combined characterization approach with DCT as the initial characterization step and topotomography (Ludwig et al., 2007) or dark-field microscopy (Simons et al., 2015) as a subsequent 'zoom' on individual grains and grain neighbourhoods, it will be become possible to resolve the three-dimensional configuration of these structures. This in turn will allow for studying slip transfer and slip-twin interactions, such as the one observed at the intersection at the grain boundary between grains $\mathrm{A}$ and $\mathrm{C}$ (Figs. $1 a$ and $1 b$ ), in the bulk of the material.

Last but not least it should be mentioned that part of the remaining inaccuracies might be due to non-negligible elastic distortions of the crystal lattices in the vicinity of twins. The six-dimensional framework used in this work does not account for these additional degrees of freedom and additional work is required in order to estimate how far this and other previously mentioned simplifications of our model for diffraction image formation affect the accuracy of the orientation maps.

\section{Conclusions}

The previously introduced six-dimensional framework for reconstruction of spatially resolved grain orientation maps from extended-beam near-field diffraction data has been extended and adapted to the characterization of structural materials with hexagonal crystal structure displaying intragranular orientation spreads of up to a few degrees and micrometre thickness twin lamellae as a result of plastic deformation.

The optimization of acquisition conditions and an explicit search over possible twin variants enables detection of deformation twins down to sub-micrometre initial thickness. Introducing an appropriate scaling of the diffracted intensities by structure and Lorentz factor, accounting for the finite impulse response of the high-resolution detector, and a global optimization carried out over a six-dimensional volume covering the parent and twin orientation(s) result in a clear improvement of the accuracy of the orientation maps when compared to the previously used single-orientation (threedimensional) reconstruction framework of X-ray diffraction contrast tomography. Cross-validation with EBSD data acquired on the sample surface shows good overall agreement but also some remaining degree of blurring and thickening of the twin domains in the X-ray orientation maps.

The nondestructive nature, straightforward combination with other tomographic imaging modalities (phase contrast, topotomography, dark-field microscopy) and improved time resolution compared to other three-dimensional orientation mapping techniques makes this approach a promising candidate for time-lapse observations at initial stages of plastic deformation in structural materials.

\section{APPENDIX $A$ \\ Theoretical determination of twin variants}

Twin orientations are related to the parent orientation by means of specific pairs of rotation axes and associated rotation angles.

Given the set of $N$ symmetry operators $S=\left\{\mathbf{S}_{i}\right\}$ with $i=1, \ldots, N$, in the form of unitary rotation matrices, and the rotation axis a which defines the type of axis for a given twinning mode, we obtain all the possible twinning rotation 
axes for the said mode by simply taking the unique results from the set $A=\left\{\mathbf{S}_{i} \mathbf{a}\right\}$, which will be another set called $A^{\prime}$.

If then we take the orientation matrix $\mathbf{g}_{\mathrm{p}}$ associated with the Rodrigues vector $\mathbf{r}_{\mathrm{p}}$ which is the orientation of the parent grain, the orientation matrices of the twin variants will be found by the simple operation

$$
\mathbf{g}_{i}=\mathbf{R}\left(\mathbf{a}_{i}^{\prime}, \alpha\right) \mathbf{g}_{\mathrm{p}}
$$

for each element $i$ in $A^{\prime}$, where $\mathbf{R}\left(\mathbf{a}_{i}^{\prime}, \alpha\right)$ is the rotation matrix defined by the axis $\mathbf{a}_{i}^{\prime}$ and the angle $\alpha$.

The same operation in equation (8) could be carried out in Rodrigues space by using the multiplication formula of Kumar \& Dawson (2000) for successive rotations:

$$
\begin{aligned}
\mathbf{r}_{i} & =\mathbf{r}_{\mathrm{p}} \mathbf{r}\left(\mathbf{a}_{i}^{\prime}, \alpha\right) \\
& =\frac{1}{1-\mathbf{r}_{\mathrm{p}} \cdot \mathbf{r}\left(\mathbf{a}_{i}^{\prime}, \alpha\right)}\left[\mathbf{r}_{\mathrm{p}}+\mathbf{r}\left(\mathbf{a}_{i}^{\prime}, \alpha\right)+\mathbf{r}_{\mathrm{p}} \times \mathbf{r}\left(\mathbf{a}_{i}^{\prime}, \alpha\right)\right],
\end{aligned}
$$

where the vector $\mathbf{r}\left(\mathbf{a}_{i}^{\prime}, \alpha\right)=\mathbf{a}_{i}^{\prime} \tan \frac{1}{2} \alpha$.

\section{APPENDIX $B$}

\section{Alignment of EBSD and DCT reference systems}

The DCT reconstruction and the EBSD map naturally come with different reference systems, and so their orientations are not directly comparable. In fact, in the orientation space, if the underlying crystal symmetry is not considered, the relationship that connects the same orientation expressed in two different reference systems (namely, the DCT and the EBSD reference systems) can be represented as

$$
\mathbf{g}_{\mathrm{DCT}}=\mathbf{g}_{\mathrm{EBSD}} \mathbf{g}_{\mathrm{DCT} \rightarrow \mathrm{EBSD}},
$$

where $\mathbf{g}_{\mathrm{DCT}}$ is the orientation matrix of the chosen orientation in the DCT reference system, while $\mathbf{g}_{\mathrm{EBSD}}$ is the orientation matrix of the chosen orientation in the EBSD reference system, and $\mathbf{g}_{\mathrm{DCT} \rightarrow \text { EBSD }}$ is the orientation matrix of the EBSD reference system expressed in the DCT reference system.

However, if the underlying crystal symmetry is taken into account, the following equation is then valid:

$$
\mathcal{F}\left(\mathbf{g}_{\mathrm{DCT}}\right)=\mathcal{F}\left(\mathbf{g}_{\mathrm{EBSD}} \mathbf{g}_{\mathrm{DCT} \rightarrow \mathrm{EBSD}}\right),
$$

where $\mathcal{F}: O^{3} \subseteq \mathbb{R}^{3} \rightarrow O^{3} \subseteq \mathbb{R}^{3}$ is the mapping of the orientations to the fundamental zone of the crystal orientation space. Unfortunately, while equation (10) is always invertible, equation (11) is not, unless the magnitude of the orientationspace Rodrigues vector associated with $\mathbf{g}_{\mathrm{DCT} \rightarrow \text { EBSD }}$ is small enough to make equation (11) equivalent to equation (10).

This means that for small calibrations of two reference systems it is enough to invert equation (10) as follows:

$$
\mathbf{g}_{\mathrm{EBSD}}^{-1} \mathbf{g}_{\mathrm{DCT}}=\mathbf{g}_{\mathrm{DCT} \rightarrow \mathrm{EBSD}} .
$$

But for larger misorientations between the two coordinate systems, it is important to know the sample geometry and how it is represented in those reference systems, so that an approximate relationship between the two can be found.

\section{Acknowledgements}

The authors acknowledge ESRF for providing access to inhouse research beamtime and computing infrastructure. We would further like to thank Arnas Fitzner for providing the material. NV and WL acknowledge the financial support of the French National Research Agency (ANR), project ANR 2010 BLAN 0935. MP and LN acknowledge financial support from EPSRC, UK (EP/F020910/1). KJB acknowledges the financial support of the Netherlands Organization for Scientific Research (NWO), project No. 639.072.005. The authors acknowledge COST Action MP1207 for networking support.

\section{References}

Bonnin, A., Wright, J. P., Tucoulou, R. \& Palancher, H. (2014). Appl. Phys. Lett. 105, 084103.

Candès, E. \& Romberg, J. (2005). $l_{1}$-MAGIC User's Guide, http:// statweb.stanford.edu/ candes/11magic/, pp. 1-19.

Christian, J. W. \& Mahajan, S. (1995). Prog. Mater. Sci. 39, 1-157.

Hayashi, Y., Hirose, Y. \& Seno, Y. (2015). J. Appl. Cryst. 48, 10941101.

Hertzberg, R. (1989). Deformation and Fracture Mechanics of Engineering Materials. Hoboken: Wiley.

Kumar, A. \& Dawson, P. (2000). Acta Mater. 48, 2719-2736.

Larson, B. C., Yang, W., Ice, G. E., Budai, J. D. \& Tischler, J. Z. (2002). Nature, 415, 887-890.

Li, S. F. \& Suter, R. M. (2013). J. Appl. Cryst. 46, 512-524.

Ludwig, W., Lauridsen, E. M., Schmidt, S., Poulsen, H. F. \& Baruchel, J. (2007). J. Appl. Cryst. 40, 905-911.

Ludwig, W., Reischig, P., King, A., Herbig, M., Lauridsen, E. M., Johnson, G., Marrow, T. J. \& Buffière, J. Y. (2009). Rev. Sci. Instrum. 80, 033905 .

Nervo, L. (2015). PhD thesis, University of Manchester, UK.

Poulsen, H. F. (2004). Three-Dimensional X-ray Diffraction Microscopy, Springer Tracts in Modern Physics, Vol. 205. Berlin, Heidelberg: Springer.

Poulsen, H. F. (2012). J. Appl. Cryst. 45, 1084-1097.

Reischig, P., King, A., Nervo, L., Viganó, N., Guilhem, Y., Palenstijn, W. J., Batenburg, K. J., Preuss, M. \& Ludwig, W. (2013). J. Appl. Cryst. 46, 297-311.

Sidky, E. Y., Jørgensen, J. H. \& Pan, X. (2012). Phys. Med. Biol. 57, 3065-3091.

Simons, H., King, A., Ludwig, W., Detlefs, C., Pantleon, W., Schmidt, S., Snigireva, I., Snigirev, A. \& Poulsen, H. F. (2015). Nat. Commun. 6, 6098.

Smith, G. C. (2006). J. Synchrotron Rad. 13, 172-179.

Suter, R. M., Hennessy, D., Xiao, C. \& Lienert, U. (2006). Rev. Sci. Instrum. 77, 123905.

Vaughan, G. B. M., Wright, J. P., Bytchkov, A., Rossat, M., Gleyzolle, H., Snigireva, I. \& Snigirev, A. (2011). J. Synchrotron Rad. 18, 125133.

Viganò, N., Ludwig, W. \& Batenburg, K. J. (2014). J. Appl. Cryst. 47, $1826-1840$.

Viganó, N., Tanguy, A., Hallais, S., Dimanov, A., Bornert, M., Batenburg, K. J. \& Ludwig, W. (2016). Sci. Rep. 6, 20618. 